\title{
Correlation of Retinopathy of Prematurity with Oxygen Saturation
}

\author{
Praveen $\mathrm{S}^{1}$, Waris $\mathrm{A}^{* 2}$ \\ ${ }^{1}$ Junior resident, MS Ophthalmology, Institute of Ophthalmology, JNMCH, AMU, Aligarh \\ ${ }^{2}$ Associate Professor, Institute of Ophthalmology, JNMCH, AMU, Aligarh \\ *Corresponding Author: Waris A; MS, FICO (UK), FICS (USA), FRCS (Edin), FRCS (Glassgow), VR Faculty, \\ Institute of Ophthalmology, JNMCH, AMU, Aligarh; waris_eye@yahoo.com
}

Received 08 April 2021; $\quad$ Accepted 26 May 2021;

Published 04 June 2021

\begin{abstract}
Retinopathy of prematurity (ROP) is an abnormal vascular proliferative disease of retina that affects preterm infants. It is a leading cause of childhood blindness worldwide despite improvement in neonatal care and management. Earlier ROP was found to be associated with oxygen therapy only. Now it was concluded that aetiology of ROP was multifactorial but three factors have shown significant association with ROP: low gestational age (GA), low birth weight (BW), prolonged exposure to supplementary oxygen following delivery. Several investigators reported that lower oxygen saturation targets at young post-gestational ages with increased oxygen saturation targets at older post gestational ages reduced the incidence of ROP. However previous clinical studies are not conclusive.
\end{abstract}

\section{Keywords: Retinopathy of Prematurity, Oxygen saturation, Gestational age, Birth weight}

\section{Introduction}

Retinopathy of prematurity [ROP] is a retinal neo-vascular disorder that can lead to severe vision loss ${ }^{[1]}$. It is mainly characterized by abnormal development of retinal vasculature. It is an important and preventable cause of childhood blindness ${ }^{[2]}$. Retinopathy of prematurity $[\mathrm{ROP}]$ is now recognized as a important cause of childhood blindness worldwide ${ }^{[3]}$. The causative factors of ROP is multifactorial and complex. It includes prematurity with a gestational age <32weeks and low birth weight [LBW] $<1500$ gram. Other risk factors, includes anaemia needing blood transfusion, prolonged exposure to supplementary oxygen, sepsis, apnea, comorbidities of prematurity, nutrition, and genetic factors [4]

ROP is an important cause of childhood blindness in countries, such as United States, and is also emerging as a major cause of blindness [the third epidemic]; in middle income countries

${ }^{[5]}$. In India, proportion of severe visual impairment and blindness due to ROP in children aged $0-15$ years in school for the blind is $0.2 \%{ }^{[6]}$.

\section{Pathogenesis}

The growth of retinal vessels is guided mainly by an endothelialcell specific mitogen which is vascular endothelial growth factor [VEGF], is one of the key angiogenic molecules implicated in pathogen It consists of two phases-:
Phase 1: Hyperoxia $\rightarrow$ Downregulation of VEGF $\rightarrow$ Vessel migration halt

Phase 2: Hypoxia $\rightarrow$ VEGF production $\rightarrow$ Neovascularization and fibrous proliferation.

However, by maintaining $\mathrm{SpO}_{2}$ values between $83 \%$ to $93 \%$ in immediate post gestation life, combined with strict oxygen fluctuations, it is possible to prevent the early vaso-obliterative phase and subsequent development of severe ROP.

\section{Relation between Oxygen Saturation and Retinopathy of Prematurity}

Many studies have been performed across the world to determine the effect of oxygen saturation on retinopathy of prematurity. McGregor ML et al. in $2002{ }^{[7]}$ conducted a prospective study in which a total of 229 STOP-ROP infants were compared with 136 HOPE-ROP infants enrolled during the same time period from same 15 hospital showed that HOPE-ROP infants progressed from pre-threshold to threshold ROP less often than STOP-ROP infants. It seems that $\mathrm{SpO} 2$ value at the time of pre-threshold diagnosis is a prognostic indicator for which infants may progress to severe ROP. York et al. in $2004{ }^{[8]}$ provided strong evidence that fluctuations in oxygen saturation target at several time duration upto 30 days following birth increased the odds of a preterm infant developing severe ROP. Vanderveen DK et al. in $2006{ }^{[9]}$ conducted a study in which he concluded that change in oximeter alarm parameters in 
the first weeks of life for infant with weight $<1250 \mathrm{~g}$ may decrease the incidence of prethreshold ROP. Sears JE, et al. in $2009{ }^{[10]}$ conducted a prospective study in which he concluded that higher oxygen target at older gestational age and lower oxygen target at early gestational age decreased the severity and incidence of ROP. Chen ML et al. in $2010{ }^{[11]}$ conducted a study among preterm infants with a gestational age of $<32$ weeks, found that early low and late high oxygen saturation were associated with a reduced risk of severe ROP. Hartnett ME et al. in $2013^{[12]}$ conducted a randomized multicenter study, Surfactant, Positive Airway Pressure, Pulse Oximetry Randomized Trial [SUPPORT] from the neonatal research network. They concluded that severe retinopathy occurred less frequently in survivors of the $85 \%$ to $89 \%$ saturation group [Relative Risk: 0.52 (95\% CI, 0.37-0.73; p<0.001)]. Alizadeh et al. in $2015^{[13]}$ did a cross-sectional retrospective study at Amir-al-momenin Eye Hospital, Iran concluded that oxygen therapy was not an independent risk factor for development of ROP. Le et al. in $2016^{[14]}$ found the incidence of ROP in their study conducted at NICU of a tertiary care hospital located in Hyderabad as $2.3 \%$.

\section{Conclusion}

Retinopathy of prematurity is a preventable eye disorder. Studies have shown that lower oxygen saturation target in early postnatal weeks and high oxygen saturation target at PMA of more than or equal to 32 weeks was associated with a decreased risk of ROP. Using a pulse oximeter and blender to provide the required oxygen dose for that target are simple and effective approaches in reducing ROP risk. We feel that a large randomized clinical trial with long term developmental follow up is required to determine the effect of oxygen saturation in ROP.

\section{List of abbreviations}

Retinopathy of prematurity (ROP)

Low birth weight (LBW)

Gestational age (GA)

Supplemental Therapeutic Oxygen for Prethreshold Retinopathy of Prematurity (STOP-ROP)

High Oxygen Percentage in Retinopathy of Prematurity (HOPEROP)

Surfactant, Positive Airway Pressure, Pulse Oximetry Randomized Trial (SUPPORT)

\section{Conflicts of Interest}

The authors declare that there is no conflict of interest regarding the publication of this paper.

\section{Funding Statement}

The authors received no specific funding for this work.

\section{Authors' contributions}

PS updated the literature with the help of PubMed and wrote the manuscript with inputs from corresponding author (WA) all authors read and approved the final manuscript.

\section{References}

[1] Christina G. Anderson, William EB, Ashima Madan. Retinopathy of prematurity and pulse oximetry: A National survey of recent practices. Journal of perinatology .2004;24:164-168

[2] Yang CS, Chen SJ, Lee FL, Hsu WM, Liu JH. Retinopathy of prematurity: screening, incidence and risk factors analysis. Zhonghua Yi Xue Za Zni Chin Med J Free China Ed. 2001 Dec;64(12):706-12

[3] Leah A. Owen, M. Elizabeth Hartnett. Current concepts of oxygen management in retinopathy of prematurity. 2014 Jan; 9(1):94-100.

[4] KIM SJ, Alexander DP, Reyan Sewan: Retinopathy of prematurity: A review of risk factors and their clinical significance: 2018 Sep-Oct. Vol 63 (5): 618-37.

[5] Andrea Zin, Glen A. Gole: Retinopathy of prematurityIncidence today. Clin Perinatol 2013(40):185-200.

[6] Gilbert C, Rahi J, Eckstein M, O’Sullivan J, Foster A. Retinopathy of prematurity in middle income countries. The Lancet. 1997 Jul 5, 350(9070):12-4.

[7] McGreogar Don L, Bremer, Cynthia Cole, Richard E. Mclead, Dale L. Retinopathy of prematurity outcome in infants with prethreshold ROP and oxygen saturation $>94 \%$ in room air: The high oxygen percentage in ROP study. Paediatrics Sep 2002, vol 110 (3):615-6.

[8] York JR, Landers S, Kirby RS, Arbogast PG, Penn JS. Arterial oxygen fluctuation and retinopathy of prematurity in very-low-birth-weight infants. J Perinatol.2004;24:82-7.

[9] Vanderveen DK, Theresa AM, Eric C. Eichenwald. Lower oxygen saturation alarm limits decrease the severity of Retinopathy of Prematurity. J AAPOS. 2006 Oct; 10(5):382-3.

[10] Sears SJ, Jeffrey pietz, Christine sonnie, David Dolcini, George Hoppe. A change in oxygen supplementation can decrease the incidence of Retinopathy of prematurity. March 2009, 116(3); 513-18.

[11] Minghua LC, Lei Guo, Lois E.H. Smith, Christiane E.L. Dammann, Olaf Damman: High or low oxygen saturation and severe ROP: A Meta-analysis. June 2010, 125(6).

[12] Hartnett ME, Lane RH. Effect of oxygen on the development and severity of Retinopathy of Prematurity. June 2013:17(3); 229-34.

[13] Alizadeh Y, Zarkesh M, Moghadam RS, Esfandiarpour B, Behboundi H, Karambin MM, et al. Incidence and risk factors for Retinopathy of Prematurity in north of Iran. J Ophthalmic Vis Res. 2013; 10(4): 424-8.

[14] Le. Retinopathy of Prematurity: Incidence, Prevalence, Risk factors, and Outcomes at a tertiary care centre in Telangana.2016: Vol 4(3): 119-122. 